\title{
Méta-matériaux et invisibilité (partie 2)
}

\author{
Sébastien GUENNEAU', Ross McPHEDRAN ${ }^{2}$, Claude AMRA' et Stefan ENOCH ${ }^{1}$ \\ 'Institut Fresnel, UMR CNRS 7249, École Centrale Marseille, Aix-Marseille-Université, \\ Campus Universitaire Saint-Jérôme, Marseille 13390 \\ ${ }^{2}$ CUDOS, School of Physics, University of Sydney, Sydney, New South Wales 2006, Australia
}

Dans la première partie de cet article, parue dans le numéro 63 de Photoniques, nous avons abordé l'invisibilité par modification d'impédance et l'invisibilité par réfraction, avant de décrire les techniques de modification d'espace appliquées aux ondes hydrodynamiques et sismiques. Nous allons, dans cette seconde partie, nous attacher à présenter ce que réalise la réfraction négative.

\section{Au détour de la lentille parfaiite}

Toutes les approches de l'invisibilité présentées dans la première partie de cet article n'exploitent pas la voie de la réfraction négative, qui a suscité un engouement rare à l'issue de débats passionnés générés par des controverses sur des paradigmes électromagnétiques. II est souvent convenu que l'inventeur de la lentille plate convergente est le russe Victor Veselago en 1968. Cette lentille plate d'indice de réfraction $n=-1$ a été revisitée et popularisée par le physicien anglais John Pendry en 2000, pour mettre au premier plan un pouvoir de résolution a priori illimité.

Or, la lentille de Pendry a d'autres atouts, comme révélé par une équipe australienne composée de l'un d'entre nous et de deux co-auteurs Nicolae Nicorovici (université de Sydney) et Graeme Milton (université d'Utah). Leur publication dans la revue Physical Reviews en 1994 contient en effet des idées phares pas entièrement appréciées à l'époque par les lecteurs et les auteurs eux-mêmes. On peut par exemple y voir une préfiguration du prototype de lentille haute-résolution de l'équipe de Zhang en 2005. Contrairement à la lentille plate de Veselago, la lentille annulaire proposée en 1994 donne lieu à un effet que l'on pourrait qualifier d' $\mathrm{d}^{\text {" ho- }}$ méopathique ». En effet, plus le cœur de la lentille est petit, plus l'effet de grossissement est important, ce qui ne peut être envisagé avec la lentille plate de Veselago.

Concernant la lentille parfaite de Pendry, celle-ci permettrait a priori d'obtenir une résolution sans limite théorique, notam- ment grâce à l'amplification des ondes évanescentes qui permettent à la lumière de jouer les passe-muraille, un peu à l'instar des tamis à photons du physico-chimiste norvégien de l'université de Strasbourg, Thomas Ebbesen, qui s'appuient sur l'existence de spoof plasmons [1], un néologisme dû à John Pendry probablement en clin d'œil au réalisme magique de Gabriel Garcia Marquez, que l'on pourrait traduire par plasmons chafouins. Une lentille convergente convexe classique d'indice de réfraction supérieur à celui du vide produira une image (inversée) d'un objet dont les détails inférieurs à une demi-longueur d'onde ne seront pas résolus. Cette barrière que l'on croyait infranchissable avant le concept de la lentille parfaite est le pendant de l'inégalité d'Heisenberg en mécanique quantique. Si l'on poursuit cette analogie, on comprendra mieux que la découverte de Pendry ait soulevé une

\section{Repliement de l'espace, réfraction négative et lentille parfaite}

Dans le cas d'un repliement de l'espace comme schématisé sur la partie gauche de la figure, les tenseurs de permittivité $\varepsilon$ et de perméabilité $\mu$ prennent des valeurs négatives. Il en résulte que l'indice de réfraction (réel) est lui aussi négatif. Le paradigme de la réfraction négative est la lentille plate de Veselago pour laquelle les images sont confondues avec leur source dans l'espace virtuel. II résulte par ailleurs du repliement de l'espace que tout objet placé dans le voisinage de la lentille doit être quasi-invisible vu qu'il est réduit à une ligne (partie droite de la figure) dans l'espace physique.

Principe de la lentille parfaite, où la région d'indice négatif permet un repliement de l'espace sur lui-même de sorte que le plan objet et le plan image sont confondus. La projection des images 1 et 2 coïncide avec celle de la source sur l'axe $x$ (haut). En effet, le chemin optique qui mesure la distance parcourue par la lumière vaut $n_{1} d / 2+n_{2} d+n_{1} d / 2=-d / 2+d-d / 2=0$, $d$ 'après le principe de Pierre de Fermat (bas).

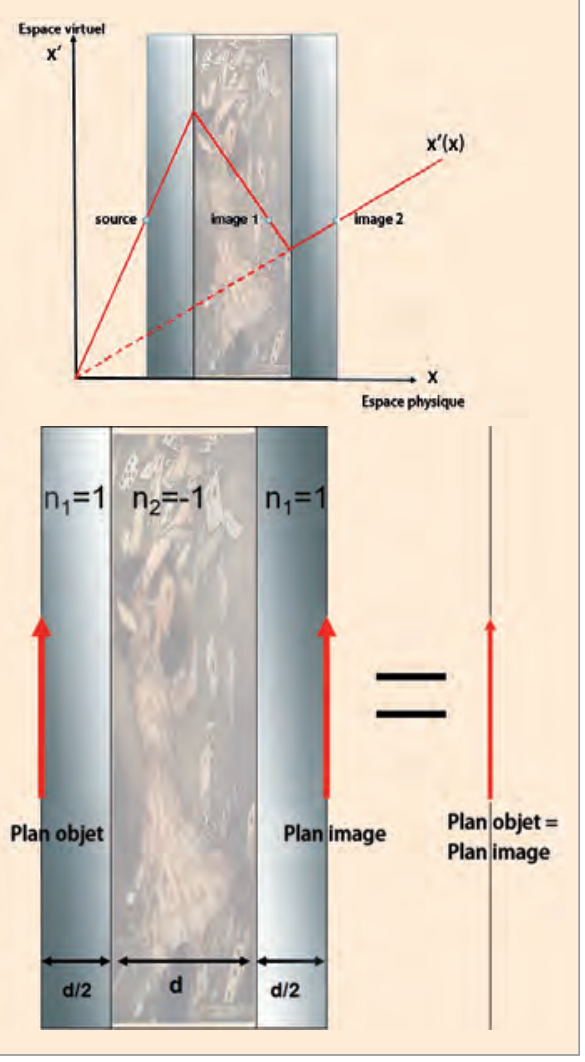


controverse à l'aune de l'émergence des méta-matériaux. Dans les faits, les métaux, pierre angulaire des méta-matériaux, sont fortement absorbants aux longueurs d'ondes optiques, ce qui limite la résolution de la super lentille de Pendry. Toutefois en 2005, une équipe de l'université de Los Angeles, dirigée par le professeur Zhang, a démontré expérimentalement une résolution supérieure à un cinquième de longueur d'onde à travers un méta-matériau constitué d'un fin film d'argent pris en sandwich entre deux couches de semi-conducteur pour une fréquence dans le spectre du visible [2]. Depuis lors, une course effrénée à la réalisation de super lentilles s'est engagée, avec des résolutions atteignant aujour$d^{\prime}$ 'hui un vingtième de longueur d'onde dans le proche infrarouge.

La réalisation d'un tel composant pour l'optique constitue un défi technologique que de nombreux groupes cherchent à relever. Mais cette lentille a encore d'autres atouts : elle permet en outre d'envisager une autre forme d'invisibilité [3] développée dans le paragraphe suivant et l'encadré.

\section{Invisibilité par réaction}

\section{(permittivité et perméabilité ont des nombres négatifs)}

En 2006, un article co-signé par Graeme Milton et Nicolae Nicorovici paraît dans les comptes rendus de l'académie des sciences anglaise (Proceedings of the Royal Society). Cet article rapporte le comportement insolite d'une excitation lumineuse au voisinage d'un cylindre de permittivité négative, mais pas nécessairement d'indice négatif (un cylindre de métal fait l'affaire pour contrôler la polarisation transverse électrique du champ électromagnétique, ce que Pendry appelle la lentille de l'indigent). Les chercheurs australiens, un peu à l'instar de Mr Jourdan avec sa prose, y prennent conscience de la portée et des enjeux des résultats préfigurés dans l'article publié en 1994 (déjà évoqué) dans la revue Physical Reviews. Non seulement cet article publié il y a presque vingt ans prophétise un type de lentille
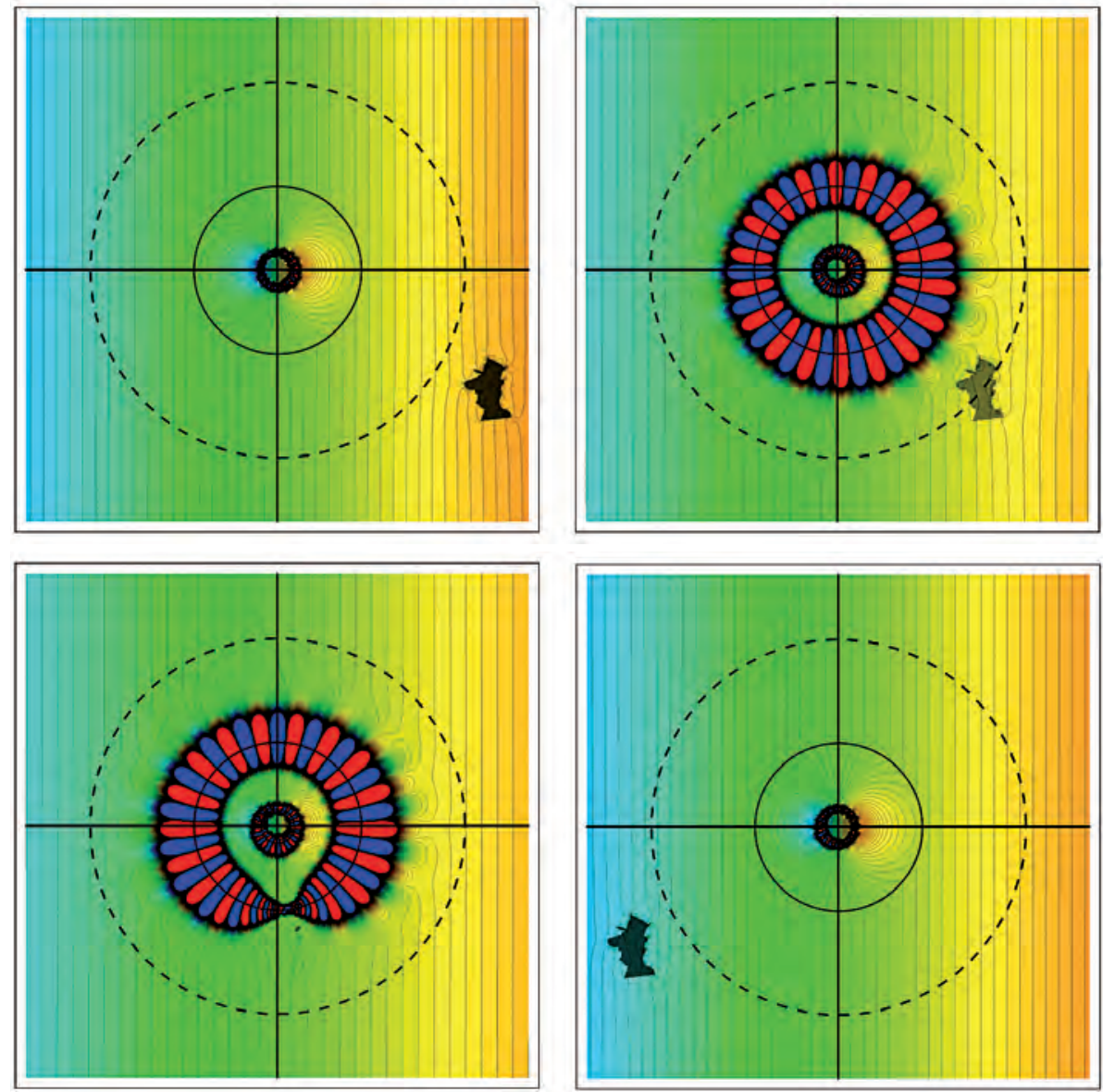

Figure 1. Invisibilité par réaction. Simulation numérique montrant le profil d'un homme d'état célèbre rendu invisible quand il approche par la droite une lentille cylindrique parfaite (en haut à gauche). La lentille réagit à la présence de l'intrus (un ensemble de dipôles électriques) par une résonance indiquée par les lobes rouges et bleus, ce qui a pour effet de perturber localement le champ électromagnétique, alors que les lignes de champ en dehors de la zone d'invisibilité (cercle en traits pointillés) ne sont pas perturbées. L'individu devient transparent en pénétrant dans la zone d'invisibilité (en haut à droite), disparaît ensuite (en bas à gauche) avant de réapparaître (en bas à droite) derrière la lentille quand il sort de la zone d'invisibilité. Simulations de Nicolae Nicorovici, CUDOS, Sydney University.

parfaite avec grossissement (contrairement à la lentille plate qui produit l'image fidèle d'un objet non renversée et de même taille), mais il ouvre une autre voie vers l'invisibilité. Dans ce cas de figure, l'objet à rendre invisible est à l'extérieur de la cape ! En fait sous certaines conditions de résonance entre la source et le cylindre, la lentille de l'indigent (figure 1) réagit à l'excitation du champ en présence d'un dipôle en l'effaçant, ce qui présente des analogies avec le principe d'équilibre chimique d'Henry Le Chatelier. Comme on peut s'en convaincre au vu de la figure 1, en théorie cela fonctionne pour un ensemble dénombrable de dipôles, pour une longueur d'onde précise et pour une cape homogène de permittivité ou d'indice de réfraction égal à -1 .

Pour comprendre cette forme d'invisibilité, on peut là encore utiliser l'analogie avec les changements de métrique induits par la présence de corps massifs dans la théorie de relativité générale de Einstein : comme noté par John Pendry, considérer un indice négatif revient à introduire de l'espace optique négatif (voir encadré). C'est d'ailleurs le principe qui sous-tend la lentille parfaite. Les physiciens utilisent ici, en théorie, un cylindre d'indice négatif pour faire disparaître un objet caché non pas dans le cylindre mais... à l'extérieur ! Ils réalisent tout simplement qu'une excitation placée près de 

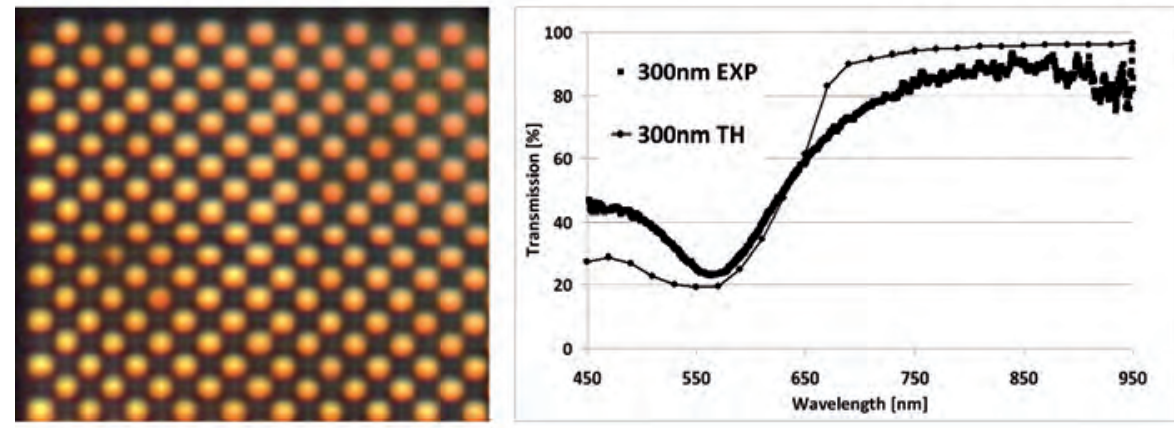

Figure 2. Transmission de la lumière blanche à travers un échiquier plasmonique constitué d'un film d'or et de quartz de $200 \mathrm{~nm}$ d'épaisseur et de 12,5 um de côté. Ce tamis à photons agit comme un filtre pour la couleur jaune-orangée (à gauche), avec une transmission extraordinaire de la lumière de 650 à $950 \mathrm{~nm}$ (à droite) (expérimental : Anantha S. Ramakrishna, Indian Institute of Technology, Kanpur ; simulation numérique: Stefan Enoch, Institut Fresnel).

la surface, comme une petite bougie, induit une réaction électromagnétique du méta-matériau, qui en retour « souffle » la bougie et la rend invisible. Harry Potter disparaît devant sa cape et pas dessous... "C'est une idée ingénieuse mais elle ne fonctionne que pour de très petits objets", constate John Pendry. Mais il n'en reste pas moins que la matière est discrète et constituée d'un grand nombre de dipôles. On peut donc arguer que même si ce type de cloaking se limite à un ensemble dénombrable de dipôles, il permettrait de rendre « presque invisible » un objet dont la réponse électromagnétique sera analogue à celle d'un ensemble de dipôles. Et même si la mise en œuvre de ce type d'invisibilité nécessite d'obtenir un indice (réel) négatif proche de -1 (pour l'heure un réel défi technologique), la compréhension profonde de son mécanisme est d'intérêt académique.

\section{Échiquiers plasmoniques et transmission extraordinaire}

Ce type d'invisibilité par réaction se décline aussi en structures de type échiquiers dont les cases alternent des indices positifs et négatifs. Une approche optogéométrique de ces échiquiers plasmoniques, où les cases d'indice négatif permettent un repliement de l'espace sur lui-même à l'instar des origamis, conduirait à penser qu'une source placée dans une case de l'échiquier engendre instan- tanément une infinité d'images parfaites. Du point de vue de l'optique géométrique, tout se passe comme si la lumière avançait à rebours dans les cases d'indice négatif. Mais ce paradoxe est résolu en notant que la lumière est intrinsèquement de nature ondulatoire dans les structures résonantes. Le comportement insolite des rayons lumineux n'est qu'une illusion, car la physique du cloaking par réaction se situe au-delà de l'horizon d'un modèle scalaire. L'Institut Fresnel poursuit actuellement cette voie qui est à la fois
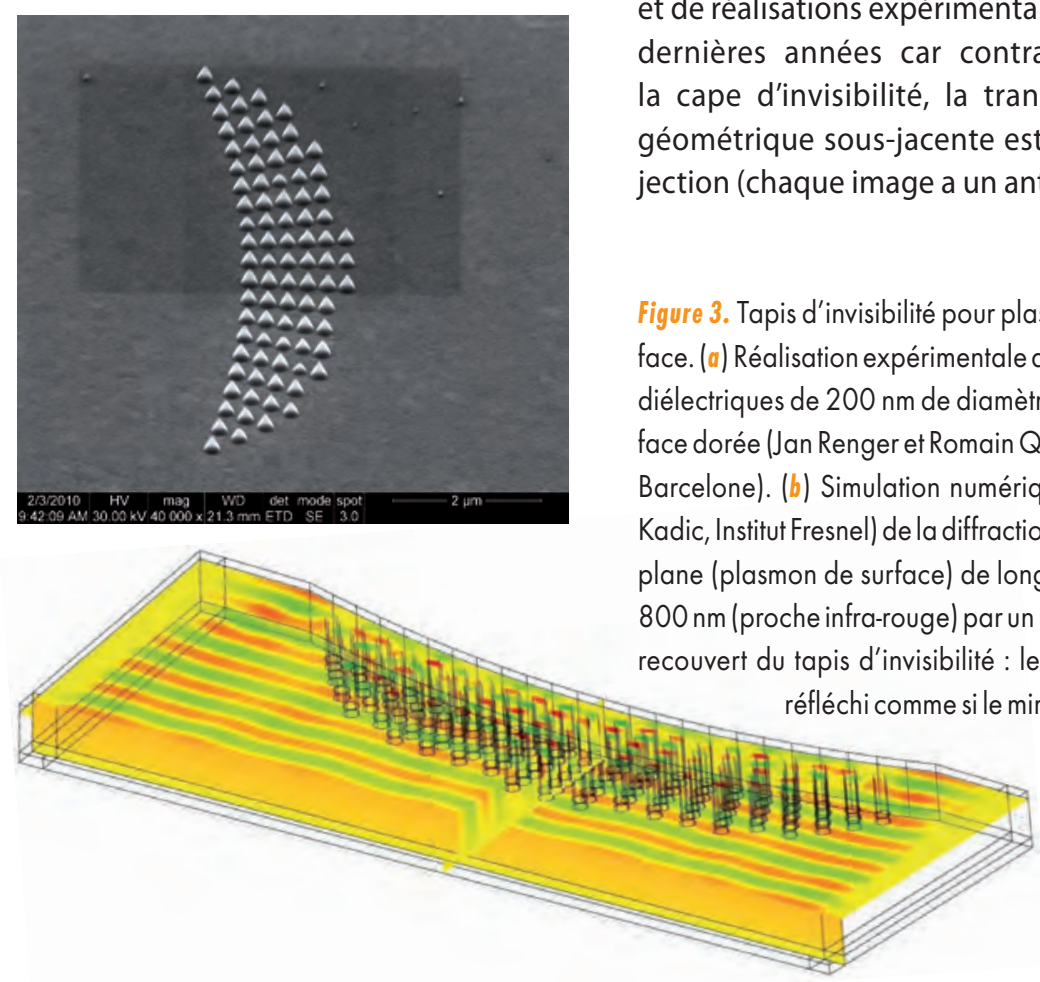

prometteuse pour le piégeage de la lumière et pour la transmission extraordinaire par le truchement de la réfraction négative des plasmons de surface dans les échiquiers d'Alice [1], en collaboration avec l'équipe du physicien Anantha Ramakrihna à I'Institut Indien de Technologie de Kanpur (IITK) (figure 2).

\section{Tapis d'invisibilité et mimétisme}

Les plasmons de surface peuvent être eux-mêmes les acteurs principaux pour des expériences de contrôle des trajectoires lumineuses. L'Institut Fresnel a par exemple démontré en partenariat avec I'ICFO Barcelone (groupe de Romain Quidant) [4] que l'on peut rétablir le front d'onde d'un plasmon de surface à l'interface d'une plaque dorée surmontée de petits plots diélectriques de diamètre $200 \mathrm{~nm}$ (figure 3). II s'agit en fait d'un tapis $d^{\prime}$ invisibilité qui recouvre un miroir cour be de telle sorte que les plasmons de surface sont réfléchis comme à l'interface d'un miroir plan. On parle alors de mimétisme plutôt que d'invisibilité. Cette nouvelle voie vers un contrôle accru des ondes a été ouverte par Jensen Li et John Pendry dans un article publié dans Physical Review Letters en 2008. Elle a donné lieu à pléthore d'études théoriques et de réalisations expérimentales ces trois dernières années car contrairement à la cape d'invisibilité, la transformation géométrique sous-jacente est ici une bijection (chaque image a un antécédent et

Figure 3. Tapis d'invisibilité pour plasmons de surface. (a) Réalisation expérimentale avec des plots diélectriques de $200 \mathrm{~nm}$ de diamètre sur une surface dorée (Jan Renger et Romain Quidant, ICFO Barcelone). (b) Simulation numérique (Muamer Kadic, Institut Fresnel) de la diffraction d'une onde plane (plasmon de surface) de longueur $\mathrm{d}^{\prime}$ onde $800 \mathrm{~nm}$ (proche infra-rouge) par un miroir courbe recouvert du tapis d'invisibilité : le plasmon est réfléchi comme si le miroir était plan. 
un seul par l'application), ce qui évite de facto toute singularité dans les tenseurs de permittivité et perméabilité. Le design des méta-matériaux s'en voit grandement simplifié.

\section{Perspectives}

\section{Vers de nouveaux types de méta-matériaux}

Les mathématiciens Allan Greenleaf, Yaroslav Kurylev, Matti Lassas et Gunther Uhlmanndes des universités de Rochester (USA), Loughborough (UK), Washington (USA), et Helsinki (Finlande), ont récemment proposé de composer la transformation géométrique de Pendry avec une seconde application qui étire la cape sphérique suivant une direction de l'espace et de la tordre de sorte à obtenir un " tunnel invisible » entre deux régions de l'espace. Ce nouveau type de méta-matériaux ouvre la voie à des circuits optiques intégrés, guides d'ondes ou endoscopes qui ne perturbent pas le champ électromagnétique ambiant. Les applications potentielles résident dans des télécommunications sécurisées ou des appareils de mesure dont le mode opératoire ne perturbe pas en retour la mesure effectuée, sans parler de furtivité. Cette extension originale de l'idée de Pendry s'appuie sur une analogie avec des singularités de l'espace-temps découvertes en 1935 par les physiciens Einstein et Rosen : des puits gravitationnels de densité et de courbure d'espace-temps infinis, plus connus sous le nom de trous de ver, sont solutions des équations de la relativité générale. Mathématiquement, cela se traduit dans les équations de Maxwell par des tenseurs de permittivité et de perméabilité qui prennent des valeurs infinies sur le bord intérieur de la cape, ce qui induit en retour une singularité sur la métrique de l'espace optique dans la théorie de Pendry. Einstein et Rosen suggéraient que de telles singularités pouvaient mener à d'autres régions de l'espace et du temps. Comme la variable temporelle n'est pas ble qui permet aux photons de se déplacer sans être vus, ce qui n'implique aucun don d'ubiquité. Deux d'entre nous ont appliqué le concept de trous de ver aux plasmons de surface.

\section{La superposition de l'effet mirage}

L'un d'entre nous a étudié par ailleurs une autre étrangeté optique avec André Nicolet et Frédéric Zolla de I'Institut Fresnel, et en collaboration avec John Pendry de I'Imperial College of London. Une source de lumière placée au sein de la cape d'invisibilité par réfraction subit l'effet de la transformation géométrique et apparaît délocalisée et déformée pour un observateur extérieur. Cet effet mirage n'est pas sans rappeler le phénomène de Fata Morgana qui s'offrait aux yeux des croisés en mer méditerranée quand des couches d'air chaud et des couches d'air froid se superposent, entraînant une succession de mirages supérieurs et de mirages inférieurs. Les images qui parviennent à l'œil de l'observateur sont ainsi

Figure 4. Avancées récentes en métamatériaux acoustiques. Les ondes de pression sont contrôlées par un tapis d'invisibilité réalisé par l'équipe de Steven Cummer de la Duke University en perforant des tranches de plexiglas (ci-contre) et elles sont focalisées avec une super résolution à l'aide d'un réseau de cannettes de soda par l'équipe de Geoffroy Lerosey et Mathias Fink de I'Institut Langevin de I'ESCPI Paris (ci-dessous). amplifiées et déformées de manière spectaculaire, et celui-ci peut alors apercevoir des objets d'aspect fantasmagorique comme des châteaux se reflétant dans la brume entre l'Italie et la Sicile. Les croisés attribuaient ce phénomène à la Fée Morgane (Fata Morgana en italien), qui, d'après la légende arthurienne, avait le pouvoir d'élever des palais au-dessus des flots. Le tapis d'invisibilité de Li et Pendry n'est d'ailleurs rien d'autre qu'un effet mirage [3].

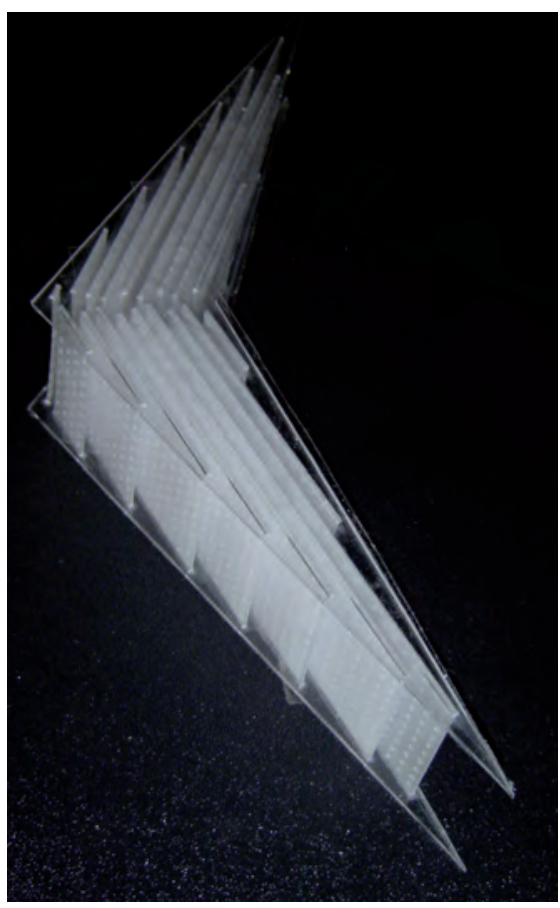

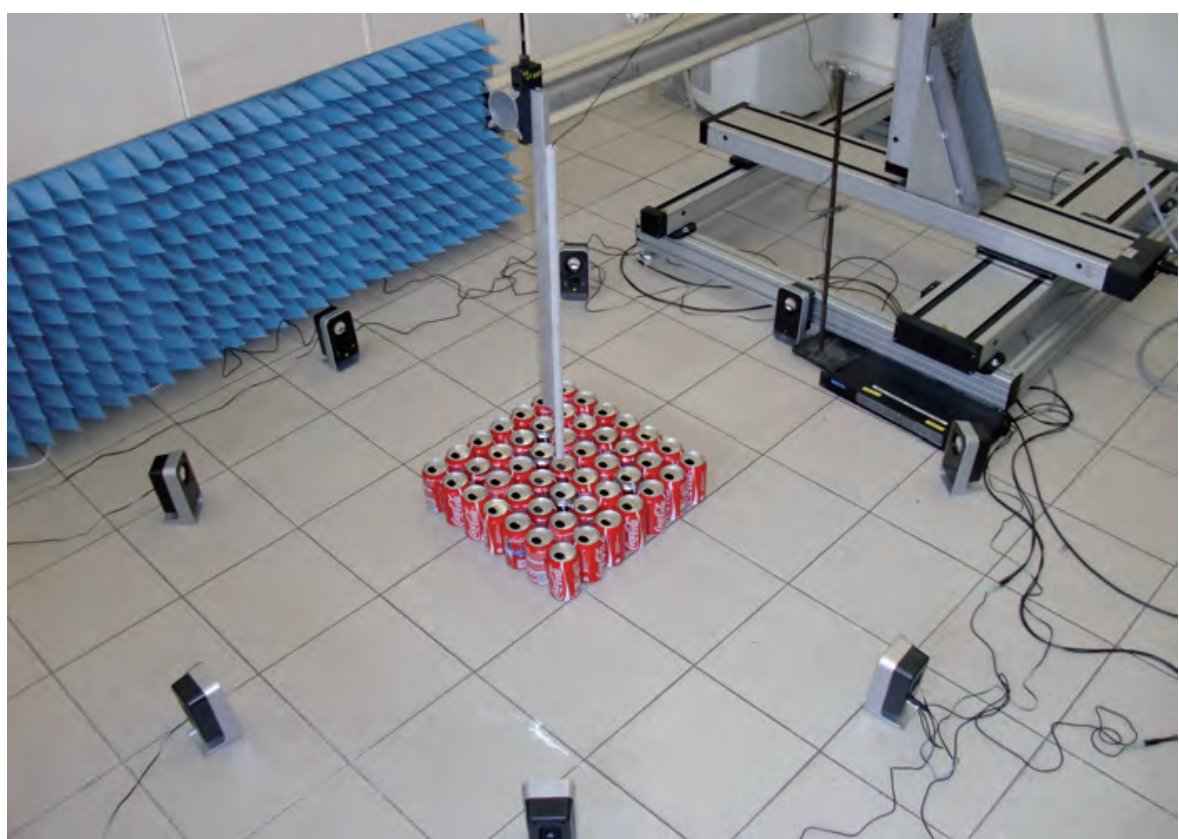




\section{Les méta-matériaux acoustiques}

Mais les illusions d'optique telles que l'invisibilité et les mirages, ainsi que la super résolution sont peut-être à chercher ailleurs : des avancées expérimentales récentes en méta-matériaux acoustiques par les groupes de Geoffroy Lerosey et Mathias Fink à I'Institut Langevin de Paris et David Cummer à l'université de Duke aux États-Unis (figure 4) suggèrent que le contrôle accru du son est en passe de prendre l'ascendant sur celui de la lumière avec notamment une résolution de l'ordre d'un vingtième de longueur d'onde pour une lentille acoustique plate avec des cannettes de soda et un petit tour de passe-passe (le retournement temporel) ! La nanotechnologie de pointe rejoint ainsi la pataphysique pour les métamatériaux soniques... Jensen Li et Xiang Zhang ont par ailleurs réalisé une hyper lentille acoustique qui pourrait aussi servir dans le cadre de l'invisibilité par réaction de manière similaire à la figure 1 .

\section{Vers le contrôle des flux de chaleur}

D'autres équations de la physique préservent par ailleurs leur forme par transformation géométrique, comme l'équation de la chaleur qui régit les phénomènes thermiques (figure 5). Deux groupes de recherche ont déjà validé expérimentalement ces prédictions (Harvard University, Karlsruher Institut für Technologie) dans le cas d'une conduction statique puis transitoire, ce qui laisse augurer d'un contrôle accru des flux de la chaleur dans les années à venir, par des voies similaires à celles ouvertes par l'avènement des méta-matériaux [2] pour le son et la lumière.

\section{Des techniques complémentaires}

Ainsi, les avancées récentes de la recherche sur les méta-matériaux, tant sur le plan théorique que technologique, sont telles que bon nombre de physiciens estiment que des capes d'invisibilité par réfraction ou par réaction vont être réalisées pour le spectre visible d'ici une dizaine d'années. D'ailleurs, les premiers prototypes de tapis d'invisibilité large bande dans le visible ont été fabriqués. Leur avènement pourrait bouleverser le monde de l'optique. Les réalisations technologiques de lentilles parfaites ou de l'indigent et de
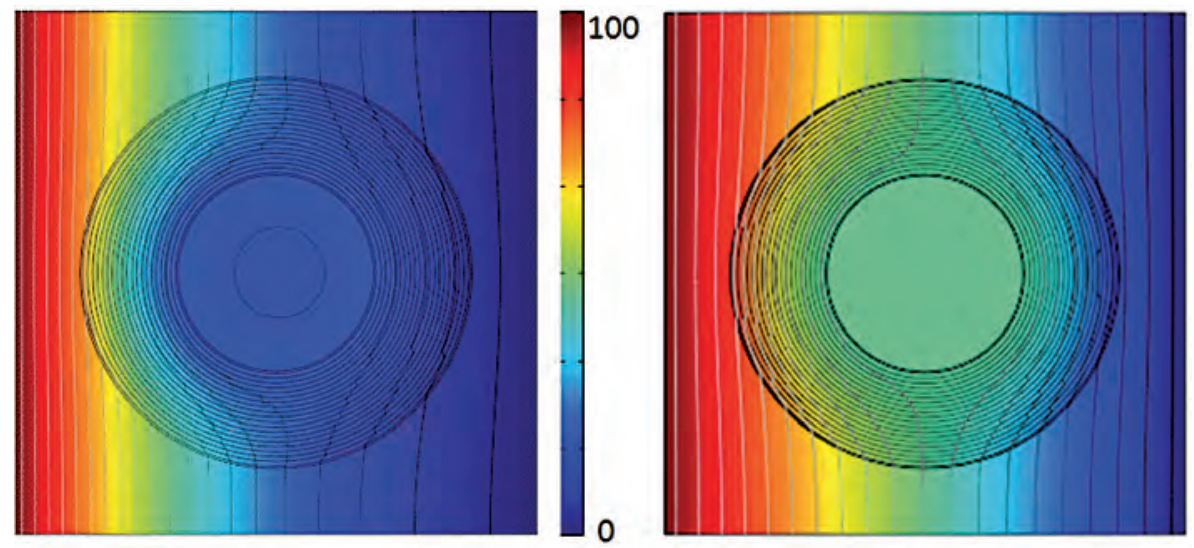

Figure 5. Vers les métamatériaux thermiques. Une source de chaleur diffuse de la gauche vers la droite. La structure multicouche est conçue pour retrouver en sortie les isothermes qui existeraient en milieu homogène. À gauche : carte de champ de la température aux temps courts (régime transitoire). À droite : carte de champ de la température aux temps longs (régime permanent) (Institut Fresnel/CNRS/École Centrale Marseille/Aix-Marseille Université).

méta-matériaux qui présentent la propriété de tordre l'espace optique sont à ce jour à un stade embryonnaire. Mais les avancées récentes en méta-matériaux acoustiques sont telles que des chapes de silence pour améliorer par exemple I'acoustique de salles de concert pourraient voir le jour à court terme. Le concept de chape d'invisibilité pour les ondes mécaniques dans les plaques a été validé expérimentalement en 2012 par l'équipe de Martin Wegener au Karlsruher Institut für Technologie (KIT) et par Nicolas Vandenberghe à I'IRPHE (CNRS/ École Centrale Marseille/Aix-Marseille Université). Dans la foulée, les premières réalisations de méta-matériaux sismiques structurés à l'échelle du mètre, ont vu le jour dans les régions grenobloise et lyonnaise en 2012, grâce à l'équipe de Stéphane Brûlé de l'entreprise Ménard (filiale du groupe Vinci). Un tapis d'invisibilité dans un canal à houle de plus de 15 mètres de long a été validé expérimentalement par l'équipe de Bernard Molin à I'IRPHE en 2012. Des prototypes de métamatériaux thermiques sont à l'étude en Allemagne (KIT), aux États-Unis (Harvard University) et en France (IEMN Lille). Une cape d'invisibilité pour des champs magnétostatiques a été proposée cette an née. Il paraîtrait donc bien téméraire de négliger l'une ou l'autre des routes vers l'invisibilité : les approches de l'invisibilité par réfraction ou réaction se révéleront certainement complémentaires suivant le type d'application envisagée. Les plus sceptiques admettront que ces deux mécanismes de cloaking présentent des paradigmes qui leur sont propres et par là même justifient un effort de recherche académique. Les plus optimistes verront dans chaque approche la pierre de touche à une révolution en marche en optique et en acoustique : la physique de transformation promet des applications fascinantes en détection radar, sonar et protection des ondes hydrodynamiques et sismiques pour l'invisibilité par réfraction, et des applications en optoélectronique pour la plasmonique pour l'invisibilité par réaction. Le mathématicien Gottfried Leibniz, fondateur du calcul infinitésimal avec Newton, conclurait « II y a de l'apparence qu'on tirera un jour des conséquences bien utiles de ces paradoxes, car il n'y a guère des paradoxes sans utilité ».

\section{Références}

[1] S. Anantha Ramakrishna et al., Plasmonic interaction of visible light with gold nanoscale checkerboards, Physical Review B 84, 245424 (2011)

[2] S. Guenneau et B. Gralak, Une optique classique sens dessus dessous, Les dossiers de La Recherche 38, 32 (2010)

[3] S. Guenneau et al., The colors of cloaks, Journal of Optics 13, 024014 (2011)

[4] J. Renger et al. Hidden progress : broadband plasmonic invisibility, Optics Express 18, 15757 (2010) 